\title{
Microscopía fototérmica para el estudio de transformaciones de fase
}

\author{
Facundo Zaldivar Escola ${ }^{1,2}$, Oscar Eduardo Martínez ${ }^{1,2}$, Nelly Mingolo $^{1}$
}

\begin{abstract}
${ }^{1}$ Universidad de Buenos Aires, Facultad de Ingeniería, Paseo Colón 850, 1063, Buenos Aires, Buenos Aires, Argentina e-mail: facundozaldivar@gmail.com,oem2005@gmail.com,nmingol@fi.uba.ar

${ }^{(2)}$ Universidad de Buenos Aires, CONICET, Facultad de Ingeniería, Paseo Colon 850, 1063, Buenos Aires, Buenos Aires, Argentina.
\end{abstract}

\section{RESUMEN}

Se presenta el desarrollo de un novedoso sistema que permite el mapeo con alta resolución espacial de la difusividad térmica en función de la temperatura hasta 1800K. El sistema se basa en una técnica fototérmica recientemente desarrollada por el grupo, consistente en la medición con un láser de prueba de la curvatura inducida por el calentamiento con un láser modulado. La utilización de tecnología de fibras ópticas provee una alta robustez al equipo. Un horno para microscopio permite realizar rampas a velocidad controlada para el estudio de transiciones de fase y la medición de la difusividad térmica en función de la temperatura. Se pueden establecer mesetas para hacer barridos espaciales con resolución microscópica para el estudio de la evolución de las fases o hacer tratamientos térmicos in situ para estudiar luego la distribución espacial de las fases presentes. En este trabajo se presenta un ejemplo, donde la señal es colectada para un punto fijo y para una dada frecuencia de modulación, en función de la temperatura de la muestra. Se diseña para ello un sistema de corrección de enfoque que compensa la deriva térmica generada por la expansión térmica del sistema. El desplazamiento lateral es corregido utilizando un algoritmo de correlación y la imagen de la cámara. Este dispositivo fue utilizado para determinar la transición de fase de una muestra testigo. Estas capacidades se muestran a partir de la determinación de la energía de activación de la transformación de fase amorfo-cristal de una aleación de Fe-B-Si.

Palabras clave: transformaciones de fase, técnica fototérmica, difusividad térmica, microscopía.

\begin{abstract}
A novel system allowing a high spatial resolution mapping of the thermal diffusivity as a function of temperature up to $1800 \mathrm{~K}$ is presented. The system is based on a photothermal technique recently developed by the group, consisting in measuring, with a probe laser beam, the curvature induced by local heating with a modulated pump laser. A microscope heating stage was added to perform temperature ramp and soak that can be used for phase transition and thermal diffusivity determinations as functions of temperature. Spatial scans with microscopic resolution can be performed to study phases evolution at constant temperature for in situ thermal treatments and subsequent study of the spatial phase distribution. In this work an example is presented where the signal at a fixed point and constant modulation frequency is collected as a function of the temperature of the sample. A focus correction device was added to compensate for the thermal drift due to the thermal expansion of the system. The lateral displacement was corrected using a cross correlation algorithm and the camera image. This device was used to determine phase transition temperature of a test sample. These capabilities are shown with the determination of the activation energy for the phase transformation glasscrystal of a Fe-B-Si alloy.
\end{abstract}

Keywords: phase transformation, photothermal technique, thermal diffusivity, microscopy.

\section{INTRODUCCIÓN}

El estudio de propiedades mecánicas y termodinámicas requiere del desarrollo de técnicas específicas que permitan la discriminación entre superficie y volumen, la determinación de gradientes de propiedades o la identificación de fases metaestables. Si bien es posible identificar la presencia de las distintas fases metaestables por medio de técnicas de difracción de rayos $\mathrm{X}$ a incidencia rasante, la caracterización microestructural 
es un problema que tales técnicas no resuelven. El bajo contraste óptico y electrónico entre las fases metaestables generadas hace necesario el desarrollo de técnicas muy sofisticadas de estudio, basadas en el revelado químico seguido de microscopía de fuerza atómica [1]. Tales técnicas son engorrosas, demandantes de equipos caros, lentas y sólo permiten explorar superficies muy planas y pequeñas.

El sistema propuesto se basa en una técnica fototérmica desarrollada por el grupo. El detalle de dicha técnica puede consultarse en [2].

\section{MATERIALES Y MÉTODOS}

\subsection{La técnica}

El sistema desarrollado consiste en caracterizar la curvatura de la superficie producto de la expansión térmica, que corresponde a la respuesta del material al aumento de temperatura provocado por la absorción de un haz laser de bombeo modulado. Un haz de prueba que emerge de una misma fibra monomodo, cuantifica el grado de curvatura superficial a través del análisis de la potencia reinyectada en dicha fibra luego de reflejarse en la superficie de la muestra estudiada. El hecho de que ambos haces viajen por la misma fibra hace que resulten inherentemente colineales, evitando problemas asociados a la estabilidad de puntería.

En trabajos previos se realizaron mapeos de estructuras proveyendo incluso información de la dureza en aceros [3]. Se demostró la capacidad de la técnica a temperatura ambiente para el estudio de pastillas sinterizadas de combustible nuclear. A partir de mapas de difusividad térmica con resolución espacial micrométrica se logró determinar la homogeneidad, distinguir óxidos interdifundidos de la mezcla de óxidos, reconocer aglomerados, poros y determinar la abundancia relativa de las diferentes especies y su distribución espacial $[4,5]$.

En esta nueva etapa se incorpora un horno para microscopio que permite realizar rampas a velocidad controlada para el estudio de transiciones de fase y la medición de la difusividad térmica en función de la temperatura hasta $1800 \mathrm{~K}$. Esta incorporación requirió del diseño de un sistema de control de foco para compensar la deriva térmica vertical así como el desarrollo de un sistema de visión automática para "tracking" de la muestra para la deriva lateral. La utilización de tecnología de fibras ópticas provee una alta robustez al equipo.

\subsection{Muestras estudiadas}

Para mostrar la potencialidad de la técnica fototérmica presentada, se estudia la transición vidrio-cristal para una aleación de $\mathrm{Fe}_{78} \mathrm{~B}_{13} \mathrm{Si}_{9}$ amorfa. Dicha muestra es una cinta de $25 \mu \mathrm{m}$ de espesor, fabricada con la técnica de Melt-Spinning.

Las aleaciones metálicas amorfas son aleaciones que no presentan orden de largo rango. Corresponden a estados metaestables del sólido, presentando cambios por debajo de la temperatura de cristalización, tales como separación de fases, difusión de varias especies y relajaciones estructurales.

Los vidrios metálicos cristalizan mediante procesos de nucleación y crecimiento similares a los que ocurren durante la solidificación convencional de metales y aleaciones justo por debajo de su punto de fusión. El estudio de la estabilidad y los mecanismos de relajación en aleaciones metálicas amorfas requiere la determinación de la temperatura de cristalización. La determinación de la estabilidad de la fase y la energía de activación de la transición se realiza a partir de la cinética de la transformación, esto es, por el retardo temporal hasta que ocurre la transformación una vez superada la temperatura de transición.

Un gran número de reacciones en fases sólidas de aleaciones metálicas pueden ser descriptas empíricamente por la ecuación de Avrami [6], que modela la fracción de volumen transformada en función del tiempo y la energía de activación que controla el proceso, que se determina realizando recocidos isotérmicos para determinar los cambios de la fracción transformada con el tiempo.

Una extensión de este método es presentada en [7] para reacciones heterogéneas, que se aplica al caso de aleaciones metálicas. En este caso, la energía de activación puede determinarse realizando rampas de calentamiento con distintas velocidades, donde se analiza la variación de la temperatura de transición $\left(T_{P}\right)$ en función de la velocidad de la rampa de temperatura $(\beta)$. Luego, mediante el gráfico de Augis-Bennett, que consiste en graficar la función $\ln \left(\beta /\left(T_{P}-T_{0}\right)\right)$ vs. $1 / T_{P}$ se obtiene una recta de pendiente $E / R$ a partir de la cual se puede determinar la energía de activación de la transición. $\mathrm{T}_{0}$ es la temperatura de inicio de la misma. .

Para la caracterización fototérmica la cinta fue seccionada en cuadrados de $5 \mathrm{~mm}$ de lado. Para relajar las tensiones provocadas durante el corte, todas las muestras fueron recocidas en su fase amorfa durante 30 minutos, a una temperatura de $573 \mathrm{~K}$. 


\subsection{Procedimiento experimental}

Un esquema de la configuración experimental se muestra en la Figura 1. Se utiliza un láser de diodos que emite a $660 \mathrm{~nm}$ para el bombeo modulado y otro a $785 \mathrm{~nm}$ para la detección (haz de prueba). Ambos son combinados por un multiplexor divisor de longitudes de onda (WDM) en una única fibra monomodo, garantizando la colinealidad. El retorno por la fibra del láser de prueba es dirigido, por medio de un circulador, a un detector conectado a un amplificador lock-in que da la amplitud y el retardo de fase de la señal fototérmica a la frecuencia de modulación. El sistema es acoplado a un microscopio metalográfico comercial por el puerto de cámara lateral. El otro puerto de cámara permite obtener una imagen de la superficie de la muestra y seleccionar, por medio del programa de control desarrollado, la región que se desea explorar. La posición de la muestra puede ser controlada en las direcciones del plano de cámara y en la perpendicular mediante una platina motorizada. El controlador del horno utilizado responde rápidamente a cambios en la temperatura sobre la muestra dada la baja inercia del horno para muestras de menos de $120 \mathrm{mg}$. La inyección de argón mejora la estabilidad del horno pero el flujo debe ser limitado a $60 \mathrm{cc} / \mathrm{min}$, para evitar la pérdida de exactitud en la medición de la temperatura. El gas atraviesa un caudalímetro y continúa su viaje hacia la cámara del horno; al salir del mismo se dirige a una trampa de agua de dos etapas que evita el ingreso de aire o agua a la cámara durante una medición. El enfriado de los sellos se realiza mediante un circuito cerrado de circulación de agua.

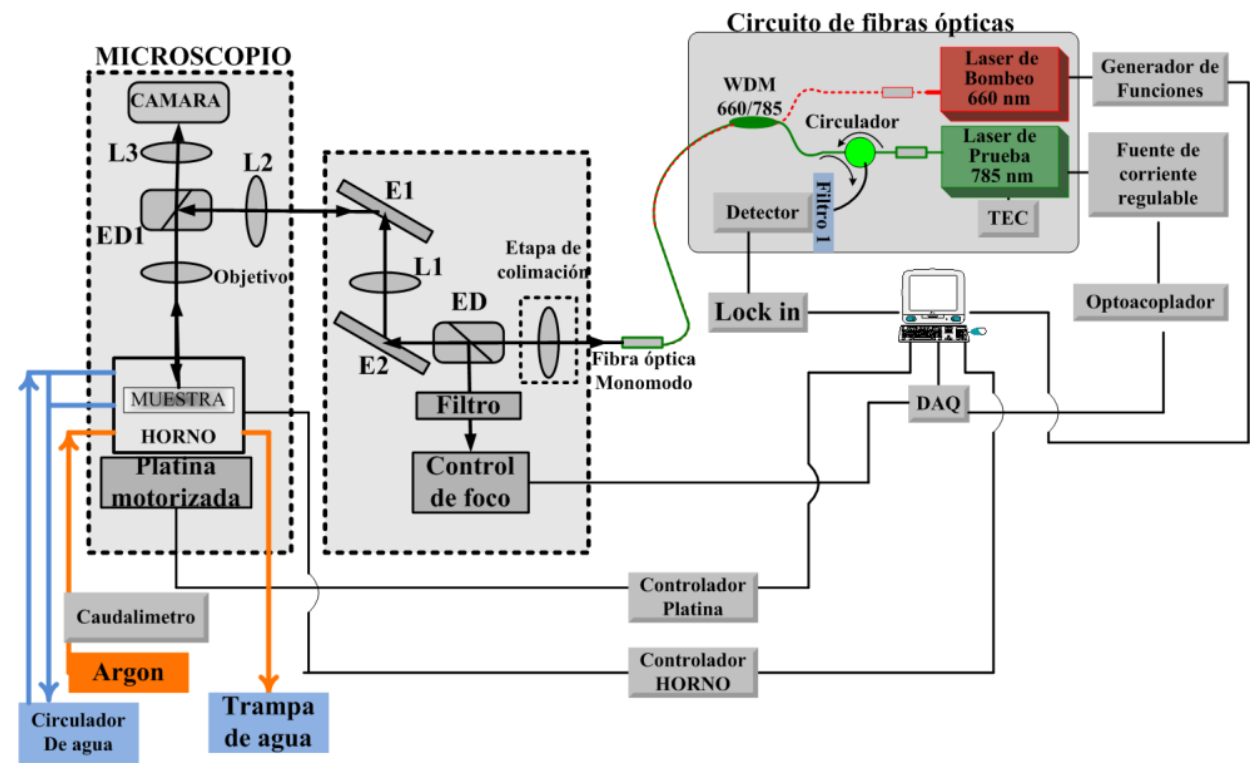

Figura 1: Esquema de la configuración experimental del microscopio fototérmico con la inclusión de un horno para microscopio. Partes: $\left(\mathrm{L}_{1}\right)$ lente de barrido, $\left(\mathrm{L}_{2}\right.$ y $\left.\mathrm{L}_{3}\right)$ lentes de tubo, $\left(\mathrm{ED}_{1}\right)$ espejo dicroico, $\left(\mathrm{E}_{1}\right)$ y $\left(\mathrm{E}_{2}\right)$ espejos, $(\mathrm{WDM})$ multiplexor divisor de longitudes de onda. (DAQ): placa de adquisición, (TEC): Refrigerador termoeléctrico.

La incorporación del horno permite el control de la temperatura de la muestra pero produce una deriva térmica tanto vertical como lateral. Para el primer caso se desarrolló un sistema de control de foco automatizado. Mediante la incorporación de un tercer haz, que emite a $405 \mathrm{~nm}$, se registra la señal que se produce en cada cuadrante de un detector de cuadro cuadrantes (4Q) por la reflexión de dicho haz sobre la muestra. La óptica interpuesta entre la muestra y el detector 4Q vuelve al haz astigmático por lo que la señal medida, generada a partir de la resta de las señales en las diagonales del detector, depende de la posición vertical de la muestra. Teniendo esta señal como referencia, se ejecuta un lazo que controla el motor para la posición vertical de forma de compensar la deriva en esa dirección.

Para la deriva lateral se hizo uso de la cámara acoplada al microscopio para tomar imágenes periódicas de la muestra a estudiar. Partiendo de una imagen inicial de referencia, el algoritmo desarrollado compara en cada paso la imagen nueva con dicha imagen, encontrando el apartamiento lateral que hace que la diferencia se minimice. Luego se ejecuta un lazo que desplaza la muestra utilizando la platina motorizada para compensar la deriva.

Las capacidades del sistema conjunto se muestran a partir de mediciones sistemáticas de la señal fototérmica sobre muestras de $\mathrm{Fe}_{78} \mathrm{~B}_{13} \mathrm{Si}_{9}$ en fase amorfa para la determinación de energías de activación y estu- 
dios de cristalización de las mismas. Las mediciones se realizaron, luego del recocido mencionado, registrando la señal fototérmica a frecuencia fija en función de la temperatura, utilizando un programa de control automático desarrollado. La frecuencia de modulación del haz de bombeo elegida para dicho barrido fue de 203 $\mathrm{KHz}$, por ser este valor cercano a la frecuencia para la cual la distancia difundida en un período de modulación se iguala al tamaño del haz (frecuencia crítica [2]). Para el objetivo utilizado (20X) se midieron los tamaños de los haces de bombeo y prueba obteniéndose $2 \mu \mathrm{m}$ y $1.7 \mu \mathrm{m}$ respectivamente.

\section{RESULTADOS Y DISCUSIÓN}

La Figura 2a muestra el resultado de la medición de la amplitud y el retardo de la fase como funciones de la temperatura, para una velocidad de calentamiento $(\beta)$ de $2 \mathrm{~K} / \mathrm{min}$. Puede observarse con mayor sensibilidad en el gráfico de amplitud que se tienen dos saltos abruptos, uno para los $760 \mathrm{~K}$ y otro para los $814 \mathrm{~K}$. Los valores obtenidos son consistentes con las temperaturas de cristalización de la muestra medidas por otra técnica en la misma cinta [8].

Adicionalmente se observó el comportamiento de la señal fototérmica luego de que la muestra fuera cristalizada. Para ello, luego de una rampa ascendente se registró la señal fototérmica enfriando la muestra. La Figura $2 \mathrm{~b}$ muestra el resultado para un caso en que la velocidad de enfriamiento fue de $8 \mathrm{~K} / \mathrm{min}$. Como puede observarse, la señal fototérmica se mantiene estable garantizando que la fase amorfa metaestable ha cristalizado completamente. En la Figura 2c se muestra la imagen de la muestra analizada.

(a)
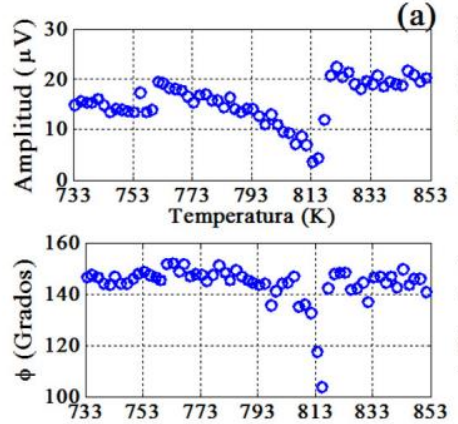

(b)

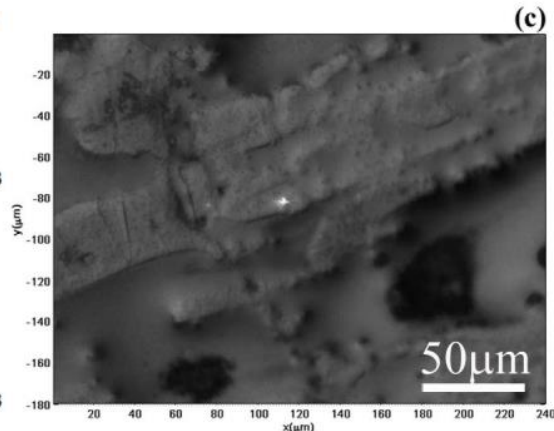

Figura 2: Resultado de la medición de amplitud y retardo de fase $\Phi$ como función de la temperatura para (a) $\beta=2 \mathrm{~K} / \mathrm{min}$ (Calentado), (b) $\beta=8 \mathrm{~K} / \mathrm{min}$ (Enfriando). (c) Imagen de una muestra de $\mathrm{Fe}_{78} \mathrm{~B}_{13} \mathrm{Si}_{9}$ de $180 \mu \mathrm{m}$ x $240 \mu \mathrm{m}$ registrada mediante la cámara del dispositivo experimental.

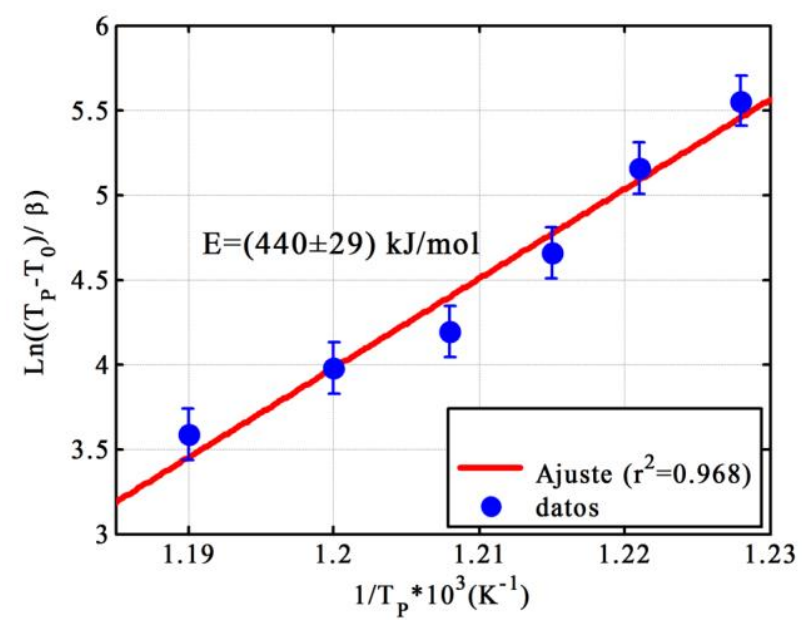

Figura 3: Gráfico de Augis-Bennett. La pendiente de la recta permite la obtención de la energía de activación de la transición.

Una vez demostrada la sensibilidad de la técnica, se realizó un proceso sistemático para la determina- 
ción de la energía de activación de la segunda cristalización. Se realizaron procesos térmicos, sobre distintos recortes de la misma cinta madre a distintas velocidades de calentamiento, midiéndose en simultaneo la temperatura, la amplitud y el retardo de fase. De esta manera pudo determinarse la dependencia de la temperatura de transición $\left(T_{P}\right)$ con la velocidad de calentamiento para $\beta=2,3,5,8,10$ y $15 \mathrm{~K} / \mathrm{min}$.

Obtenida la temperatura $T_{P}$ para cada velocidad de calentamiento, es posible realizar el grafico correspondiente de Augis-Bennet. La pendiente de la recta resultante de este gráfico permite la obtención de la energía de activación (E) de la transición, como se ilustra en la Figura 3. Dicha energía de activación resultó ser $(440 \pm 29) \mathrm{kJ} / \mathrm{mol}$. Este valor se encuentra en el rango reportado previamente $[8,9]$.

\section{CONCLUSIONES}

El estudio de este material ha permitido probar que el sistema en su desarrollo final es robusto dado que permite efectuar mediciones en condiciones críticas. Las cintas amorfas son maleables y deformables por efectos de la temperatura, mientras que cristalizadas son frágiles, sin embargo, el conjunto desarrollado permitió mantener la muestra enfocada, buscar con facilidad la región a explorar y mantenerla durante el calentamiento.

Se ha logrado determinar la dependencia de la temperatura de la transición con la velocidad de calentamiento y la energía de la transformación amorfo-cristal gracias a las capacidades del sistema de controlar la temperatura de la muestra, y corregir de forma automática el foco y la posición de los haces debido a los corrimientos que producen las deformaciones propias del material al ser calentado. Estas capacidades hacen de esta microscopía una herramienta única para la caracterización de propiedades térmicas a escala microscópica en función de la temperatura.

\section{AGRADECIMIENTOS}

Este trabajo se realizó con el subsidio Ubacyt de la Universidad de Buenos Aires

\section{BIBLIOGRAFÍA}

[1] ROS-YÁÑEZ, HOUBAERT, T. Y., MERTENS, A., "Characterization of TRIP-assisted multiphase steel surface topography by atomic force microscopy”, Materials Characterization, v. 47 pp. 93-104, 2001.

[2] MINGOLO, N., MARTÍNEZ, O. E, "Focus shift photothermal method for thermal diffusivity mapping", J. Applied Physics, v. 111, pp. 123526 - 123526-7. 2012.

[3] CROSSA ARCHIOPOLI, U., MINGOLO, N., et al, "Two-dimensional mapping of micro-hardness increase on surface treated steel determined by photothermal deflection microscopy", Surface and Coatings Technology, v. 205, pp. 3087-3092, 2011.

[4] ZALDIVAR ESCOLA, F., MARTÍNEZ, O. E, et al., "Photothermal microscopy applied to the characterization of nuclear fuel pellets"; Journal of Nuclear Materials, v. 435, , pp. 17-24, 2013.

[5] ZALDIVAR ESCOLA, F., KEMPF, R., MINGOLO, N., et al., "Characterization of Sintered Mixed Oxides by Photothermal Microscopy”; International. J. Thermophysics, v. 37, pp.1-18, 2016.

[6] AVRAMI, M. "Kinetics of Phase Change. I. General Theory", .Journal of Chemical Physics, v. 12, pp. 1103-1112, 1939.

[7] AUGIS, J.A., BENNETT, J.E., "Calculation of Avrami parameters for heterogeneous solid state reactions using a Modified Kissinger's method”, Journal of thermal analysis, v. 13, pp. 283 -292, 1978.

[8] CESA, Y., Detección Optoacústica Resonante de Transiciones de Fase en Superficie y Volumen, Tesis de doctorado FIUBA, Buenos Aires, Argentina, 2004.

[9] RAMANAN, V. R. V, FISH, G. E., "Crystallization kinetics in Fe-B-Si metallic glasses”, J. Applied Physics, v. 53, pp. 2273-2275, 1982. 